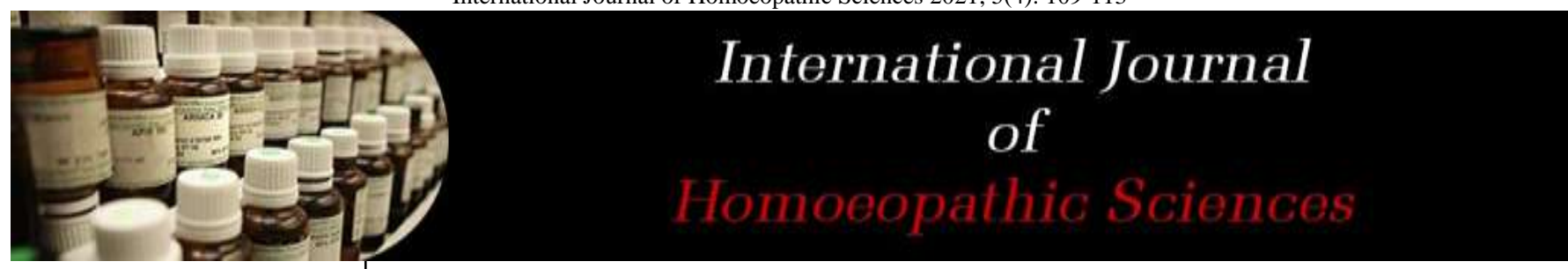

E-ISSN: $2616-4493$ P-ISSN: 2616-4485 www.homoeopathicjournal.com IJHS 2021; 5(4): 109-113 Received: 08-08-2021 Accepted: 12-09-2021

Dr. Neil Praisy Assistant Professor, Department of Practice of Medicine, MNR Homeopathic Medical College Sangareddy, Telangana, India

\section{Dr. MK Kamath}

Professor and Head, Department of Practice of Medicine, Father Muller Homoeopathic Medical College and Hospital, Mangalore, Karnataka, India
Corresponding Author: Dr. Neil Praisy Assistant Professor, Department of Practice of Medicine, MNR Homeopathic Medical College, Sangareddy, Telangana, India

\title{
Efficacy of homoeopathy in modifying the cardiac risk factors in prehypertensive individuals: A clinical study
}

\section{Dr. Neil Praisy and Dr. MK Kamath}

DOI: $\underline{\text { https://doi.org/10.33545/26164485.2021.v5.i4b.458 }}$

\begin{abstract}
Persons with prehypertension are at twice the risk of developing hypertension compared to normotensive individuals.

Objective: To evaluate the cardiac risk factors and their prevalence in patients with pre-hypertension and to determine the changes in cardiac risk factors such as diabetes, dyslipidaemia in prehypertensive individuals after homoeopathic treatment.

Methods: This is a quasi - experimental 6-month prospective study with an evaluation before and at the end of the treatment without control design.

Results: The measured ' $p$ ' value for all the parameters was 0.000 , which is less than 0.01 , that denotes a high significant reduction in SBP, DBP, FBS, TC, TG, LDL, VLDL and increase in HDL values after constitutional homoeopathic treatment.

Conclusion: This study concludes that Homoeopathy is effective in modifying the cardiac risk factors such as diabetes and dyslipidemia in prehypertension.
\end{abstract}

Keywords: Prehypertension, FBS, Lipid profile, Homoeopathy

\section{Introduction}

High blood pressure is the leading cause of cardiovascular disease (CVD) and deaths globally. It is associated with at least 7.6 million deaths per year worldwide $(13.5 \%$ of all deaths), making it the leading risk factor for CVD ${ }^{[1]}$. The importance of blood pressure (BP) as a modifiable risk factor for CVD is well recognized and many effective and inexpensive blood pressure-lowering treatments are available. The Seventh Report of the Joint National Committee (JNC VII) on Prevention, Detection, Evaluation and Treatment of High Blood Pressure [2] suggested a new classification for borderline blood pressure (BP), the "prehypertension" (PHTN) level. This new classification describes people with BPs between 120 and $139 \mathrm{~mm}$ of $\mathrm{Hg}$ systolic or 80 and $89 \mathrm{~mm}$ of $\mathrm{Hg}$ diastolic. This "new" category between normal BP and established hypertension (HTN) includes a population at considerable risk for developing HTN and in which lifestyle modifications are needed [2] The designation pre-hypertension is considered to recognize those individuals in whom early intervention by adoption of healthy lifestyle could reduce BP, declining the rate of progression of BP to hypertensive levels with age, or prevent HTN entirely. Moreover, the total morbidity, mortality and economic loss caused by cardiovascular events amongst high normal blood pressure(pre-hypertension) population far exceeds that caused by hypertensive population ${ }^{[3]}$

A prospective cohort study by Huang Y, et al. suggested that PHTN should be categorized as low range and high range as they analyzed a marked difference in the risk of CVD for BP readings between 120 to $129 / 80$ to $84 \mathrm{mmHg}$ and 130 to $139 / 85$ to $89 \mathrm{mmHg}$. This study concluded that, PHTN has a high risk of CVD even at low ranges and reaffirms it is important for a health professional to check the risk of CVD primarily ${ }^{[4]}$. A meta-analysis of cross sectional and longitudinal studies by Gou X, et al. showed that the many risk factors such as overweight, dyslipidemia, impaired glucose metabolism were observed. According to the descriptive analysis the significant factors such as old age, obesity, history of smoking and drinking were reported to be important predictors of progression to HTN ${ }^{[5]}$. In the recent years, in spite of increased awareness, prevention, detection and treatment of PHTN, it still remains an important public health challenge ${ }^{[6]}$. Jeemon P, Prabhakaran D, et al. have concluded in a sentinel surveillance study that an Indian industrial population with elevated BP showed a cluster of cardiovascular risk factors and thereby there is a significant increased 
CVD risk, so it is important for a physician to primarily screen the patients with elevated BP and elicit all other risk factors and give effective management. CVD risk reductions programs had an impact in reducing the clustering risk factors and the proportion of individuals with low risk increased ${ }^{[7]}$

PHTN may be initiated in a genetically predisposed individual by any environmental factors such as stress, salt intake and estrogen levels which affect the variables that control blood pressure ${ }^{[8]}$. So, symptoms are expressed in mind as well as in body and every individual has a different way of expressing. A quote fromDr Roberts "Principles and Art of Cure by Homoeopathy": "No two individuals are alike. The development of the vital energy in one differs from that in another. Each one possesses a special personality and a special psychophysical construction which is determined by the interplay of hereditary tendencies and factors of disease." Thus, this study is taken up to observe the changes in the BP of PHTN individuals as well as the variations of cardiovascular risk factors such as Fasting Blood Sugar (FBS) levels and serum lipid profile in the individuals treated with homoeopathic constitutional remedies.

\section{Methodology}

Research Design: This is a quasi - experimental 6-month prospective study with an evaluation before treatment and at the end of the treatment without control design. Sample size-The sample consisted of 100 cases of PHTN taken by purposive sampling method. The subjects were screened for the risk factors such as diabetes and dyslipidaemia, and 49 cases fulfilling inclusion criteria were selected for the study.

Source of data: This study was conducted on patients who attended the Out Patient Department (OPD) and peripheral centres of Father Muller Homoeopathic Medical College and Hospital, Deralakatte and Kankanady, Mangaluru.

Period of Study: The study was conducted on the cases available from May 2018 - February 2020.

Inclusion Criteria: Patients aged from 20-80 years, of both sexes. Family history of HTN. Pre-hypertensive individuals with systolic pressure between $120-139 \mathrm{mmHg}$ and diastolic pressure between 80-89 $\mathrm{mmHg}$, Diabetics with FBS $>100 \mathrm{mg} / \mathrm{dL}$, Dyslipidaemia TC $>200 \mathrm{mg} / \mathrm{dL}$; TG $>150$ $\mathrm{mg} / \mathrm{dL} ; \mathrm{HDL}<45 \mathrm{mg} / \mathrm{dL}, \mathrm{LDL}>100 \mathrm{mg} / \mathrm{d}$ Land VLDL > 34 $\mathrm{mg} / \mathrm{dL}$.

Exclusion Criteria: Subjects who are under medication for HTN and dyslipidaemia. Subjects who have a previous history of CVD. Subjects with kidney diseases, adrenal problems or thyroid dysfunction.

\section{Materials and Method}

100 cases of PHTN were screened and 49 cases which satisfied the above criteria were selected for the study. Data was collected from subjects by interviewing and clinical examination, after obtaining a written consent. BP was measured using a standard mercury sphygmomanometer by calculating the average of three consecutive readings at separate visits with an interval of at least one week. Individuals who fall under the pre-hypertensive category i.e. SBP between 120-139 mmHg and DBP between 80-89
mmHg were screened for the risk factors by checking their FBS and Lipid profile. Patients who were diabetic [FBS $>100 \mathrm{mg} / \mathrm{dL}$ ] and dyslipidaemia [TC $>200 \mathrm{mg} / \mathrm{dL}, \mathrm{TG}>150$ $\mathrm{mg} / \mathrm{dL}, \mathrm{HDL}<45 \mathrm{mg} / \mathrm{dL}, \mathrm{LDL}>100 \mathrm{mg} / \mathrm{dL}$ and VLDL > $34 \mathrm{mg} / \mathrm{dL}$ ] were included in the study. The data was recorded in the Standardized Case Record (SCR) of Father Muller Homoeopathic Medical College and Hospital, analysed, totality was erected. After reference to the Homoeopathic Materia Medica and Repertory was made as required by the case, suitable remedy with suitable potency and dose was administered. After following up the cases the inferences were drawn by analysis of the outcome. In the course of treatment 9 cases dropped out for various reasons, and 40 cases were assessed after 6 months, at the end of the study period. There were no control groups used in the study and all the subjects were treated on outpatient basis.

Follow Ups: Patients were followed for a period of 6 months from the commencement of treatment. BP was checked in every follow-up. Assessment of effectivenessEach patient was evaluated after every follow up for the changes in BP to understand the improvement. At the end of the study i.e., after 6 months, FBS and lipid profile was evaluated, and changes were analysed. The remedies prescribed were all manufactured at Father Muller Homoeopathic Pharmaceutical Division and dispensed at OPD of Father Muller Homoeopathic Medical College and Hospital, Mangalore and its allied peripheral centres.

Statistical Analysis: The collected data was analysed by Mean, Standard deviation, paired' $t$ ' test and chi square/Fishers exact test.

\section{Research Hypothesis}

Homoeopathic constitutional treatment is effective in modifying the cardiac risk factors such as diabetes and dyslipidaemia in PHTN.

Null Hypothesis: Homoeopathic constitutional treatment is not effective in modifying the cardiac risk factors such as diabetes and dyslipidaemia in PHTN.

\section{Results and Observation}

A total of 100 pre-hypertensive individuals were screened, out of which 49 subjects had risk factors such as diabetes and dyslipidaemia. The individual's BP was checked in every follow up and FBS and lipid profile investigations were sent before and after 6 months of treatment. The following observations were made.

Table 1: Distribution of cases according to age group

\begin{tabular}{|c|c|c|}
\hline Age Group & Frequency & Percent \\
\hline $20-30$ & 12 & $12 \%$ \\
\hline $31-40$ & 21 & $21 \%$ \\
\hline $41-50$ & 31 & $31 \%$ \\
\hline $51-60$ & 24 & $24 \%$ \\
\hline Above 60 & 12 & $12 \%$ \\
\hline Total & 100 & $100 \%$ \\
\hline
\end{tabular}

In the subjects screened between 20-80 years, $12 \%$ were pre-hypertensive in the age group 20-30 years, $21 \%$ between the age group $31-40$ years, $31 \%$ between the age group 41 50 years, $24 \%$ between the age group 51-60 years and $12 \%$ above 60 years. 


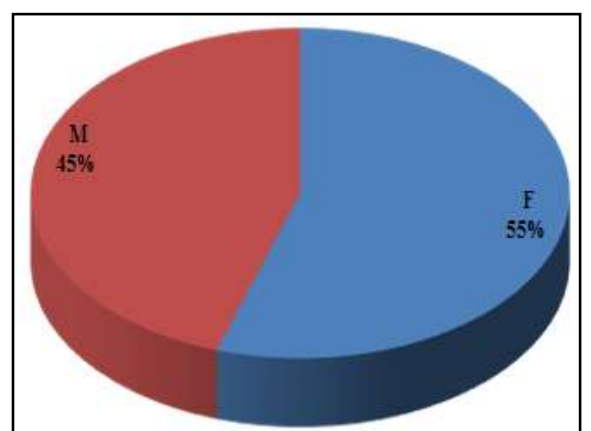

Fig 1: Distribution of cases based on gender

There were 55 females and 45 males among the 100 pre- hypertensive individuals

Table 2: Prevalence of risk factors

\begin{tabular}{|c|c|c|c|}
\hline Risk Factor & Risk & Count & Column N \% \\
\hline \multirow{3}{*}{ FBS } & No risk & 70 & $70.00 \%$ \\
\cline { 2 - 4 } & Risk & 30 & $30.00 \%$ \\
\cline { 2 - 4 } & Total & 100 & $100.00 \%$ \\
\hline \multirow{3}{*}{ LIPID } & No risk & 63 & $63.00 \%$ \\
\cline { 2 - 4 } & Risk & 37 & $37.00 \%$ \\
\cline { 2 - 4 } & Total & 100 & $100.00 \%$ \\
\cline { 2 - 4 } & Total & 100 & $100.00 \%$ \\
\hline
\end{tabular}

In the 100 prehypertensive subjects it was observed that 30 $\%$ were diabetic and $37 \%$ with dyslipidaemia.

Table 3: Analysis of SBP before and after treatment

\begin{tabular}{|c|c|c|c|c|c|c|c|c|c|}
\hline Parameter & & Mean & Std. Deviation & Change (\%) & Mean Difference & S.D of difference & T value & p & \\
\hline \multirow{2}{*}{ Systolic } & Pre & 131.70 & 3.06 & 5.92 & 7.80 & 5.65 & \multirow{2}{*}{8.73} & 0.000 & HS \\
\cline { 2 - 10 } & Post & 123.90 & 6.93 & & & & & & \\
\hline
\end{tabular}

The mean of SBP before treatment was 131.70 with a standard deviation of 3.06.The mean of SBP after treatment was 123.90 with a standard deviation of 6.93 with mean difference of before and after treatment7.80 and the mean change $5.92 \%$. The calculated ' $t$ ' value was 8.73 and ' $p$ ' value was 0.000 which is less than 0.01 which denotes a high significant reduction in SBP before and after treatment.

Table 4: Analysis of DBP before and after treatment

\begin{tabular}{|c|c|c|c|c|c|c|c|c|c|}
\hline Parameter & & Mean & Std. Deviation & Change (\%) & Mean Difference & S. d of Difference & T Value & p & \\
\hline \multirow{2}{*}{ Diastolic } & Pre & 84.70 & 2.95 & \multirow{2}{*}{6.02} & \multirow{2}{*}{5.10} & \multirow{2}{*}{4.51} & \multirow{2}{*}{7.16} & \multirow{2}{*}{0.000} & \\
\hline & Post & 79.60 & 4.53 & & & & & & \\
\hline
\end{tabular}

The mean of DBP before treatment was 84.70 with a standard deviation of 2.95. The mean of DBP after treatment was 79.60 with a standard deviation of 4.53 with mean a difference of before and after treatment 5.10 and the mean change $6.02 \%$. The calculated ' $t$ ' value was 7.16 and ' $p$ ' value was 0.000 which is less than 0.01 which denotes a high significant reduction in DBP before and after treatment.

Table 5: Analysis of FBS levels pre and post treatment

\begin{tabular}{|c|c|c|c|c|c|c|c|c|c|}
\hline Parameter & & Mean & Std. Deviation & Change (\%) & Mean difference & S.d of difference & T value & p & \\
\hline \multirow{2}{*}{ FBS } & PRE & 111.48 & 23.32 & \multirow{2}{*}{17.99} & 20.06 & 17.12 & 7.41 & 0.000 & HS \\
\cline { 2 - 10 } & POST & 91.43 & 19.20 & & & & & & \\
\hline
\end{tabular}

The mean of FBS levels before treatment was 111.48 with a standard deviationof 23.32. The mean of FBS after treatment was 91.43 with a standard deviation of 19.20 with mean difference of before and after treatment20.06and the mean change $17.99 \%$. The calculated ' $t$ ' value was 7.41 and ' $p$ ' value was 0.000 which is less than 0.01 which denotes a high significant reduction in FBS before and after treatment.

Table 6: Analysis of TC values pre and post treatment

\begin{tabular}{|c|c|c|c|c|c|c|c|c|c|}
\hline Parameter & & Mean & Std. Deviation & Change (\%) & Mean Difference & S.d of difference & T value & p & \\
\hline \multirow{2}{*}{ Tc } & Pre & 214.86 & 38.79 & 12.24 & 26.31 & 22.92 & 7.26 & 0.000 & HS \\
\cline { 2 - 10 } & Post & 188.55 & 29.73 & & & & \\
\hline
\end{tabular}

The mean of TC levels before treatment was 214.86 with a standard deviationof38.79. The mean of TC after treatment was 188.55 with a standard deviation of 29.73 with mean difference of before and after treatment 26.31 and the mean change $12.24 \%$. The calculated ' $t$ ' value was 7.26 and ' $p$ ' value was 0.000 which is less than 0.01 which denotes a high significant reduction in TC before and after treatment

Table 7: Analysis of TG levels pre and post treatment

\begin{tabular}{|c|c|c|c|c|c|c|c|c|c|}
\hline Parameter & & Mean & Std. Deviation & Change (\%) & Mean Difference & S.d of difference & t value & p & \\
\hline \multirow{2}{*}{ TG } & Pre & 154.44 & 56.15 & \multirow{2}{*}{12.67} & 19.57 & 23.88 & 5.18 & 0.000 & HS \\
\cline { 2 - 9 } & Post & 134.87 & 48.33 & & 2.57 & & & \\
\hline
\end{tabular}

The mean of TG levels before treatment was 155.44 with a standard deviationof56.15. The mean of TG after treatment was 134.87 with a standard deviation of 48.33 . With mean difference of before and after treatment were 19.57 and the mean change $12.67 \%$. The calculated ' $t$ ' value was 5.18 and 'p' value was 0.000 which is less than 0.01 which denotes a high significant reduction in TG before and after treatment. 
Table 8: Analysis of HDL levels before and after treatment

\begin{tabular}{|c|c|c|c|c|c|c|c|c|c|}
\hline Parameter & & Mean & Std. Deviation & Change (\%) & Mean Difference & S.d of difference & T value & p & \\
\hline \multirow{2}{*}{ HDL } & Pre & 43.85 & 9.65 & \multirow{2}{*}{-14.68} & -6.44 & 7.32 & 5.56 & 0.000 & HS \\
\cline { 2 - 10 } & Post & 50.28 & 9.23 & & & & \\
\hline
\end{tabular}

The mean of HDL levels before treatment was 43.85 with a standard deviationof9.65. The mean of HDL after treatment was 50.28 with a standard deviation of 9.23 . With mean difference of before and after treatment were -6.44 and the mean change- $14.68 \%$. The calculated ' $t$ ' value was 5.56 and 'p' value was 0.000 which is less than 0.01 which denotes a high significant increase in HDL before and after treatment.

Table 9: Analysis of LDL levels before and after treatment

\begin{tabular}{|c|c|c|c|c|c|c|c|c|c|}
\hline Parameter & & Mean & Std. Deviation & Change (\%) & Mean Difference & S.d of difference & T value & p & \\
\hline \multirow{2}{*}{ LDL } & Pre & 132.31 & 31.24 & 16.22 & 21.47 & 24.83 & 5.47 & 0.000 & HS \\
\cline { 2 - 10 } & Post & 110.85 & 24.57 & 16.2 & & & \\
\hline
\end{tabular}

The mean of LDL levels before treatment was 132.31 with a standard deviationof31.24. The mean of LDL after treatment was 110.85 with a standard deviation of 24.57 with mean difference of before and after treatment 21.47 and the mean change $16.22 \%$.

The calculated 't' value was 5.47 and 'p' value was 0.000 which is less than 0.01 which denotes a high significant reduction in LDL before and after treatment.

Table 10: Analysis of VLDL level pre and post treatment

\begin{tabular}{|c|c|c|c|c|c|c|c|c|c|}
\hline Parameter & & Mean & Std. Deviation & Change (\%) & Mean Difference & S.d of Difference & T value & p & \\
\hline \multirow{2}{*}{ VLDL } & Pre & 30.48 & 12.42 & 20.86 & 6.36 & 7.88 & \multirow{2}{*}{5.10} & 0.000 & HS \\
\cline { 2 - 9 } & Post & 24.12 & 9.57 & & & & & & \\
\hline
\end{tabular}

The mean of VLDL levels before treatment was 30.48 with a standard deviationof 12.42. The mean of VLDL after treatment was 24.12 with a standard deviation of 9.57 . With mean difference of before and after treatment were 6.36 and the mean change $20.86 \%$. The calculated ' $t$ ' value was 5.10 and 'p' value was 0.000 which is less than 0.01 which denotes a high significant reduction in SBP before and after treatment.

\section{Statistical Analysis}

Analysis done using paired ' $t$ ' test and chi square/Fishers exact test showed significant $p$ values with reduction of SBP, DBP, TC, TG, LDL, VLDL and increase in HDL in pre-hypertensive individuals. There is significant reduction in SBP, DBP, FBS, TC, TG, LDL, VLDL and increase in HDL values $(\mathrm{p}=.000)$ after constitutional homoeopathic treatment. Hence null hypothesis is rejected and alternative hypothesis is accepted.

\section{Discussion}

A total of 100 pre-hypertensive individuals were screened, out of which 49 subjects had risk factors such as obesity, diabetes and dyslipidaemia. In the subjects screened between $20-80$ years, $31 \%$ of individuals between the age group 41-50 years had high prevalence. This differs from the study done by Yadav $\mathrm{S}$ where highest prevalence was observed in the group aged between 30-39 years ${ }^{[9]}$. The prevalence of PHTN observed to decline with increasing age in both men and women, which is similar to a study by Yang $\mathbf{J}$ et al. ${ }^{[10]}$. There were 55 females and 45 males among the 100 pre-hypertensive individuals, yet in a study done by Grotto I et al. observed a high prevalence in males with a widespread presence across the age groups, but increased prevalence was observed in women with advancing age ${ }^{[11]}$.

In this study we observed $30 \%$ had diabetes among the 100 screened pre-hypertensive individuals which supports the study done by David et al which demonstrated individuals with PHTN have higher rates of association with diabetes [12]

This study also shows $37 \%$ individuals with dyslipidaemia which concur with the findings of a study done by Williams et al. ${ }^{[13]}$. Out of 100 individuals 51 had no risk factors and 16 individuals exhibited one of the risk factors, 18 individuals had any two risk factors and 15 individuals had all the 3 risk factors. Thus, this study observed clustering of cardiovascular risk factors such as obesity, diabetes and dyslipidaemia in pre-hypertensive individuals which is identical to observations made by Haffner SM et al. ${ }^{[14]}$.

After a statistical analysis of 40 patients using paired ' $t$ ' test the mean difference of SBP before and after treatment was 7.80 and the mean change $5.92 \%$, DBP before and after treatment was 5.10 and the mean change $6.02 \%$. FBS before and after treatment was 20.06and the mean change $17.99 \%$.TC before and after treatment was 26.31 and the mean change $12.24 \%$. TG before and after treatment was 19.57 and the mean change $12.67 \%$. HDL before and after treatment was -6.44 and the mean change-14.68\%.LDL before and after treatment was 21.47 and the mean change $16.22 \%$. VLDL before and after treatment was6.36 and the mean change $20.86 \%$.

The measured ' $p$ ' value for all the parameters was 0.000 which is less than 0.01 which denotes a high significant reduction in SBP, DBP, FBS, TC, TG, LDL, VLDL and increase in HDL values after constitutional homoeopathic treatment. This observation supports the studies done by Aboobacker $\mathrm{R}$ in which homoeopathic similimum was effective in lowering SBP and DBP in adult females ${ }^{[15]}$, and another study done by Tiwari NL et al have found Constitutional useful in the management of Diabetes Mellitus Type $2^{[16]}$. But the results observed in this study partially correspond to a study done by Ray NP et al which conclude that TC, TG and LDL were reduced after 6 months in Individualised homoeopathic (IH) patients, and no marked improvement was shown regarding HDL and VLDL [17]. 
Pre-hypertensive individuals had a higher prevalence of cardio metabolic risk factors than members of normotensive $[18,19]$. Thus, the study convincingly proves the research hypothesis (alternative hypothesis) that Homoeopathy is effective in modifying the cardiac risk factors such as obesity, diabetes and dyslipidemia in PHTN.

\section{Conclusion}

After a statistical analysis of 6 months post homoeopathic constitutional treatment, the measured ' $p$ ' value for all the parameters was 0.000 , which is less than 0.01 , which denotes a high significant reduction in SBP, DBP, FBS, TC, TG, LDL, VLDL and increase in HDL values after constitutional homoeopathic treatment. Therefore, this study concludes that Homoeopathy is effective in modifying the cardiac risk factors such as obesity, diabetes and dyslipidemia in PHTN.

\section{Acknowledgment}

I would like to acknowledge the Administrator, Principal, Viceprincipal, medical superintendent, teaching and nonteaching staff of Father Muller homeopathic medical college and hospital for their extended supported.

\section{References}

1. Lawes CM, Vander Hoorn S, Rodgers A. Global burden of blood-pressure-related disease, 2001. The Lancet 2008;371(9623):1513-1518.

2. Lenfant C, Chobanian AV, Jones DW, Roccella EJ. Joint National Committee on the Prevention, Detection, Evaluation, and Treatment of High Blood Pressure: Seventh Report of the Joint National Committee on the Prevention, Detection, Evaluation, and Treatment of High Blood Pressure (JNC 7): Resetting the hypertension sails. Hypertension 2003;41:1178-1179. PubMed.

3. Munjal YP. API Textbook of Medicine. $9^{\text {th }}$ edition. Volume I. JP Medical Ltd; 2015 Aug 30.p.686. Adi BS, Adi GB, Jamadade AK. A Comparison of the Efficacy of Gymnema Sylvestre $6 \mathrm{Ch}$ and Gymnema Sylvestre Mother Tincture in Cases of Type 2 Diabetes Mellitus. World Journal of Current Medical and Pharmaceutical Research 2020;1:133-8.

4. Huang Y, Wang S, Cai X, Mai W, Hu Y, Tang H, et al. Prehypertension and incidence of cardiovascular disease: a meta-analysis. BMC medicine 2013;11(1):177.

5. Guo X, Zou L, Zhang X, Li J, Zheng L, Sun Z, et al. Wong ND, Sun Y. Prehypertension: a meta-analysis of the epidemiology, risk factors, and predictors of progression. Texas Heart Institute Journal 2011;38(6):643-652.

6. Zhang $\mathrm{W}, \mathrm{Li} \mathrm{N}$. Prevalence, risk factors, and management of prehypertension. International journal of hypertension 2011.

7. Jeemon P, Prabhakaran D, Goenka S, Ramakrishnan L, Padmanabhan S, Huffman $\mathrm{M}$, et al. Impact of comprehensive cardiovascular risk reduction programme on risk factor clustering associated with elevated blood pressure in an Indian industrial population. The Indian journal of medical research 2012;135(4):485-493.

8. Balekundri AA. Efficacy of Homoeopathic Remedies in Essential Hypertension. [Internet]. 2012. Raj P, Sharma
PK, Ambily B. Assessing the reliable symptoms of rhus tox and kali carb in the treatment of lumbar spondylosis with sciatica syndrome-an observational study. International Journal of Alternative and Complementary Medicine 2020;30:11-6.

9. Yadav S, Boddula R, Genitta G, Bhatia V, Bansal B, Kongara $\mathrm{S}$, et al. Prevalence \& risk factors of prehypertension \& hypertension in an affluent north Indian population. Indian Journal of Medical Research 2008;128(6):712-21.

10. Yang J, Lu F, Zhang C, Liu Z, Zhao Y, Gao F, et al. Prevalence of prehypertension and hypertension in a Chinese rural area from 1991 to 2007. Hypertension Research 2010;33(4):331-7.

11. Grotto I, Grossman E, Huerta M, Sharabi Y. Prevalence of prehypertension and associated cardiovascular risk profiles among young Israeli adults. Hypertension 2006;48(2):254-9.

12. Jenkins DJ, Wong JM, Kendall CW, Esfahani A, Ng VW, Leong TC, et al. The effect of a plant-based lowcarbohydrate ("Eco-Atkins") diet on body weight and blood lipid concentrations in hyperlipidemic subjects. Archives of internal medicine 2009;169(11):1046-54.

13. Williams RR, Hunt SC, Hopkins PN, Stults BM, Wu LL, Hasstedt SJ, et al. Familial dyslipidemic hypertension: evidence from 58 Utah families for a syndrome present in approximately $12 \%$ of patients with essential hypertension. Jama 1988;259(24):357986.

14. Haffner SM, Ferrannini E, Hazuda HP, Stern MP. Clustering of cardiovascular risk factors in confirmed prehypertensive individuals. Hypertension 1992;20(1):38-45. Adi BS, Adi GB, Tanuja B. A comparison of efficacy of Plantago major and Calendula officinalis in the management of gingivitis: a randomized controlled clinical trial. International Journal of Alternative and Complementary Medicine 2020;30:1-7.

15. Aboobaker R. A comparison of the efficacy of two homoeopathic interventions in the treatment of primary hypertension in adult females (Doctoral dissertation).

16. Tiwari NL, Tamboli P. Diabetes mellitus-Defining scope and clinical approach for homoeopathic management.

17. Ray NP, Uchat U, Purkait R. An open-label randomized pilot trial to study the effectiveness of Allium sativum mother tincture $(\varnothing)$ in comparison with individualized homoeopathic medicines in treatment of dyslipidemia.

18. Gupta S, Kapoor S. Sex differences in blood pressure levels and its association with obesity indices: who is at greater risk. Ethnicity \& disease 2010;20(4):370.

19. Mainous III AG, Everett CJ, Liszka H, King DE, Egan BM. Prehypertension and mortality in a nationally representative cohort. The American journal of cardiology 2004;94(12):1496-500. 